\title{
Prevalence of diabetes distress and associated factors among patients with diabetes using antihypertensive medications in community health centres in Bandung City, Indonesia
}

\author{
Sofa Dewi Alfian ${ }^{* 1,2}$, Imam A. Wicaksono ${ }^{1,2}$, Norisca A. Putri ${ }^{3}$, Rizky Abdulah ${ }^{1,2}$ \\ ${ }^{1}$ Department of Pharmacology and Clinical Pharmacy, Universitas Padjadjaran, Faculty of Pharmacy, \\ Jatinangor, Sumedang, West Java, Indonesia \\ ${ }^{2}$ Center of Excellence in Higher Education for Pharmaceutical Care Innovation, Universitas Padjadjaran \\ Jatinangor, Sumedang, West Java, Indonesia \\ ${ }^{3}$ Faculty of Pharmacy, Department of Pharmaceutics and Pharmaceutical Technology, Universitas Padjadjaran \\ Jatinangor, Sumedang, West Java, Indonesia
}

Submitted: 21-02-2021

Reviewed: 12-06-2021

Accepted: 29-07-2021

\begin{abstract}
Diabetes distress is common among patients with type 2 diabetes mellitus (T2DM), which remains unrecognized in primary care settings. A higher level of diabetes distress was found among T2DM patients with comorbidities. The objectives of this study are to assess the prevalence rate of diabetes distress and its association with sociodemographic factors among T2DM patients using antihypertensive medication in Bandung City, Indonesia. An observational cross-sectional survey was performed in six community health centres in Bandung City, Indonesia, among T2DM patients aged at least 18 years who were using antihypertensive medications. Diabetes distress subscales (emotional, regimen, interpersonal, and physician-related distress) were evaluated using the validated Diabetes Distress Scale. Pearson $\chi 2$ and Mann-Whitney tests were performed to assess the associations of patients' sociodemographic factors (age, gender, insurance type, education, and duration since diagnosed with diabetes and hypertension) with diabetes distress. Of 105 patients who participated and completed the survey (response rate 93.8\%), most of them were female and were aged 60-69 years. A total of 38 patients (36.2\%) had moderate-high diabetes distress with emotional (56.2\%) and regimen (53.3\%) distress as the most commonly reported distress. Moderate-high emotional and regimen diabetes distress were significantly higher among the elderly (p 0.014) and patients who could not

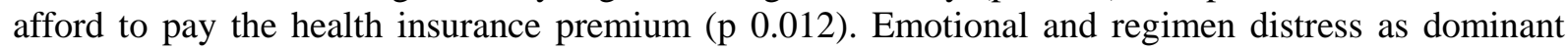
forms of diabetes distress was observed among T2DM patients using antihypertensive medications. A routine diabetes distress assessment is needed in T2DM patients with comorbidity in primary care settings.
\end{abstract}

Keywords: diabetes distress, blood pressure-lowering medication, associated factors

\footnotetext{
*Corresponding author:

Sofa Dewi Alfian

Faculty of Pharmacy, Universitas Padjadjaran

Jatinangor, Sumedang, West Java, Indonesia

Email: sofa.alfian@unpad.ac.id
} 


\section{INTRODUCTION}

Diabetes mellitus is one of the most common non-communicable diseases which is experienced by more than 400 million people around the world and expected to be the 7th cause of mortality by 2030 (Ogurtsova et al., 2017). In Indonesia, 10.3 million of patients with diabetes was reported in 2017 and this number was estimated to increase to 16.7 million of patients by 2045 (International Diabetes Federation, 2017). Management of type 2 diabetes mellitus (T2DM) requires patients to engage in regular physical activity, follow a healthy diet, monitor blood glucose levels, and take diabetes medication (American Diabetes Association, 2019a). However, incorporating these lifetime self-management plans into patients' daily life can be challenging (Carls et al., 2017).

Diabetes distress refers to the psychological and emotional responses to the burden and stress related to the management of diabetes (Polonsky et al., 2005). Different types of diabetes distress, that is, emotional, regimen, physician, and interpersonal-related distress are representing different sources of distress (Polonsky et al., 2005). About a quarter of patients experiencing diabetes distress in developed countries (Dennick et al., 2015), while this number is expected to be higher in developing countries (Chew et al., 2016). Furthermore, 17.2\% of those with no diabetes distress at the first evaluation are more likely to experience a high level of diabetes distress in the following 18 months (Fisher et al., 2009).

Diabetes distress was related to fewer self-care behaviors (Fisher et al., 2012), lower quality of life (Bruno et al., 2019; Chew et al., 2015), suboptimal glycaemic control (Fisher et al., 2010), and increased morbidity (Fisher et al., 2007). Therefore, assessing and monitoring diabetes distress is recommended as part of clinical care in developed countries (American Diabetes Association, 2019b). However, the implementation of routine assessment of diabetes distress lacks in Indonesia (Arifin et al., 2019).

T2DM patients in Indonesian primary health care experienced more distress compared to those in tertiary health care settings (Arifin et al., 2019). The lack of emotional support from family and healthcare providers as well as difficulty to manage the burden of comorbidities such as hypertension, may contribute to an increase of diabetes distress (Emre et al., 2018; Wardian and Sun, 2014). Among T2DM patients, antihypertensive can be perceived by patients as co-medication, which may give particular problems that need to be addressed. However, this problem is not well addressed when designing health interventions in primary care settings. To develop tailor intervention according to the individual patient's needs, insight into the magnitude of diabetes distress and associated determinants among T2DM patients with comorbidity is needed. The objectives of this study are to assess the prevalence rate of diabetes distress and its association with sociodemographic factors among T2DM patients using antihypertensive in Bandung City, Indonesia.

\section{MATERIALS AND METHOD}

A cross-sectional survey was performed in Bandung City, Indonesia, from October 2018 to September 2019. Patients were recruited from six purposively selected Community Health Centres (CHCs) according to a sufficient number of T2DM patients diagnosed with hypertension. CHCs are primary health care centres staffed with health care providers such as doctors, nurses, midwives, and pharmacists, which provide integrated chronic disease management programs at the sub district level. The study has been approved by the Health Research Ethics Committee of Universitas Padjadjaran No. 1137/UN6.KEP/EC/2018. Informed consent has been obtained from all patients participated in this study.

Patients aged at least 18 years, diagnosed with T2DM for more than one year, using antihypertensive medications, and literate in the Indonesian language were eligible to participate. We excluded patients who were not able to take their own medication, with severe physical or mental constraints, were pregnant, or were in the lactation period. Pharmacists at the CHC screened for patients' eligibility and informed the researcher or trained research assistant when a patient was 
eligible to explain and describe the study and to ask them to sign the informed consent statement. We asked eligible patients to complete a self-reported questionnaire independently. In some cases, however, elderly patients who could not read and/or answer the questionnaires were interviewed by trained research assistants.

Distress associated with the burdens of managing diabetes was measured using the Diabetes Distress Scale (DDS-17) (Polonsky et al., 2005). The DDS consists of 17 items evaluating the distress experienced by the patients last month using four subscales, that is, emotional-related distress (five items, e.g., "feeling angry, scared, and/or depressed when I think about living with diabetes"), physician-related distress (four items, e.g., "feeling that my doctor doesn't take my concerns seriously enough"), regimen-related distress (five items, e.g., "feeling that I am often failing with my diabetes routine), and interpersonal-related distress (three items, e.g., "feeling that friends or family do not appreciate how difficult living with diabetes can be). Patients' responses in each subscale were added and divided by the total number of items in that specific subscale. Based on clinical validation of the DDS, the total score was classified into three groups, that is, those with a total score less than 2.0 (mild distress), between 2.0 and 2.9 (moderate distress), and 3.0 (high distress) (Fisher et al., 2012). To develop tailored intervention, it is important to recognize the patients who need additional support as early as possible. Therefore, we combined patients with moderate and high distress. The DDS in the Indonesian version was reported to be valid and reliable (Arifin et al., 2017).

We collected the patient's sociodemographic factors, that is, gender, age, education completed (without formal education or elementary school, junior and senior high school, or university), and health insurance type. Health insurance type was defined as patients who could not afford to pay the health insurance premium (BPJS-PBI), patients who afford to pay the health insurance premium (BPJS-Non PBI), or patients who do not have health insurance. A structured case report form was used to record the duration of diabetes and hypertension (years).

The minimum sample size was calculated by the Slovin formula (Almeda et al., 2010). A minimum of 100 patients was required to obtain a margin error of 0.05 and a $95 \%$ confidence level.

\section{Data Analysis}

Descriptive statistics were performed to describe the patient characteristics. Pearson $\chi^{2}$ and Mann-Whitney tests were performed to evaluate the associations between patients' sociodemographic factors and diabetes distress. Since there were few missing data, we conducted complete-case analyses. SPSS software (version 25.0; IBM, Armonk, NY, USA) was used to analyze all statistical tests.

\section{RESULT AND DISCUSSION}

Of 105 patients who completed the survey with a response rate of $93.8 \%$., the majority of them were female and were aged 60-69 years (Table 1). A total of 38 patients (36.2\%) had moderate-high diabetes distress (Table 1). The prevalence rate of high diabetes distress was reported between 18 to 35\% (Abdulbari Bener, 2011; L. Fisher et al., 2009, 2010). The relatively high prevalence rate of diabetes distress in our study might be due to the combination of moderate and high diabetes distress. Although the disease onset is reported as one of the significant factors related to distress (Hannonen et al., 2015), T2DM patients are more likely to develop diabetes distress across the nature course of the disease. Therefore, to prevent the negative impacts of diabetes distress, we assume that early support is needed once patients identified with at least moderate diabetes distress.

Most of the patients reported with high-moderate emotional (56.2\%) and regimen distress $(53.3 \%)$ (Figure 1). Emotional distress was reported as the dominant form of diabetes distress among patients with T2DM as compared to other domains of distress in previous studies (Gahlan et al., 2018; Rubin and Peyrot, 1999; Zanchetta et al., 2016). The higher prevalence rate of emotional distress might be because patients perceived implementing self-management, self-care and other psychological activities as a difficult aspects. Our findings indicating support is needed to target emotional and 
regimen distress when managing diabetes distress in T2DM patients using antihypertensive medications. We further observed that the majority of patients had mild physician distress. This can be explained by the satisfaction of T2DM patients toward the physicians in the CHCs. A good relationship between patients and physicians is important for the patients' involvement in their health care, which may influence their health outcomes (Chipidza et al., 2015).

Table 1. Patient characteristics $(\mathbf{N}=\mathbf{1 0 5})$

\begin{tabular}{|c|c|}
\hline Characteristic & $\mathbf{N}(\%)$ \\
\hline \multicolumn{2}{|l|}{ Gender } \\
\hline Male & $26(24.8)$ \\
\hline Female & $79(75.2)$ \\
\hline \multicolumn{2}{|l|}{ Age in years } \\
\hline$\leq 49$ & $8(7.6)$ \\
\hline $50-59$ & $25(23.8)$ \\
\hline $60-69$ & 44 (41.9) \\
\hline$\geq 70$ & $26(24.8)$ \\
\hline Missing & $2(1.9)$ \\
\hline \multicolumn{2}{|l|}{ Type of insurance } \\
\hline BPJS-PBI & $44(41.9)$ \\
\hline BPJS-Non PBI & $52(49.5)$ \\
\hline Without insurance & $9(8.6)$ \\
\hline \multicolumn{2}{|l|}{ Last education level } \\
\hline \multicolumn{2}{|l|}{ Without formal education / } \\
\hline Junior high school & $25(23.8)$ \\
\hline Senior high school & $37(35.2)$ \\
\hline University & $9(8.6)$ \\
\hline Missing & $2(1.9)$ \\
\hline \multicolumn{2}{|l|}{ Time from diagnosis, years } \\
\hline Diabetes, mean (SD) & $4.0(3.9)$ \\
\hline Missing & 10 \\
\hline Hypertension, mean 9SD) & $5.1(5.5)$ \\
\hline Missing & 14 \\
\hline \multicolumn{2}{|l|}{ Diabetes Distress } \\
\hline Mild & $66(62.9)$ \\
\hline Moderate & $38(36.2)$ \\
\hline Missing & $1(1.0)$ \\
\hline
\end{tabular}

Abbreviations: BPJS-PBI: patients who could not afford to pay the health insurance premium, BPJS-Non PBI: patients who afford to pay the health insurance premium, SD: standard deviation

We further analyzed the subscales of diabetes distress since the average of total diabetes distress scores might be within normal ranges, yet, the distress subscales might reveal specific distress warranting more attention. We observed that moderate-high emotional and regimen diabetes distress was higher among the elderly (60-69 years) and patients who could not afford to pay the health insurance premium (Table 2). Regimen-related distress is an important area of diabetes distress that focuses on patients' treatment. It might be that elderly patients in this study may have experienced more difficulty in managing their diabetes and hypertension regimens concurrently. Furthermore, these patients may feel a lack of emotional support as well as have difficulty coping with their condition (Baek et al., 2014; Naicker et al., 2017). In addition, patients who could not afford to pay the health insurance premium. Indicating that these patients were those with low economic status. These patients might experience a higher emotional burden compared to those with high economic status. Therefore, 
a behavioral intervention which targeted on the emotional aspect of managing diabetes is needed to prevent and to reduce high diabetes distress (Sturt et al., 2015).

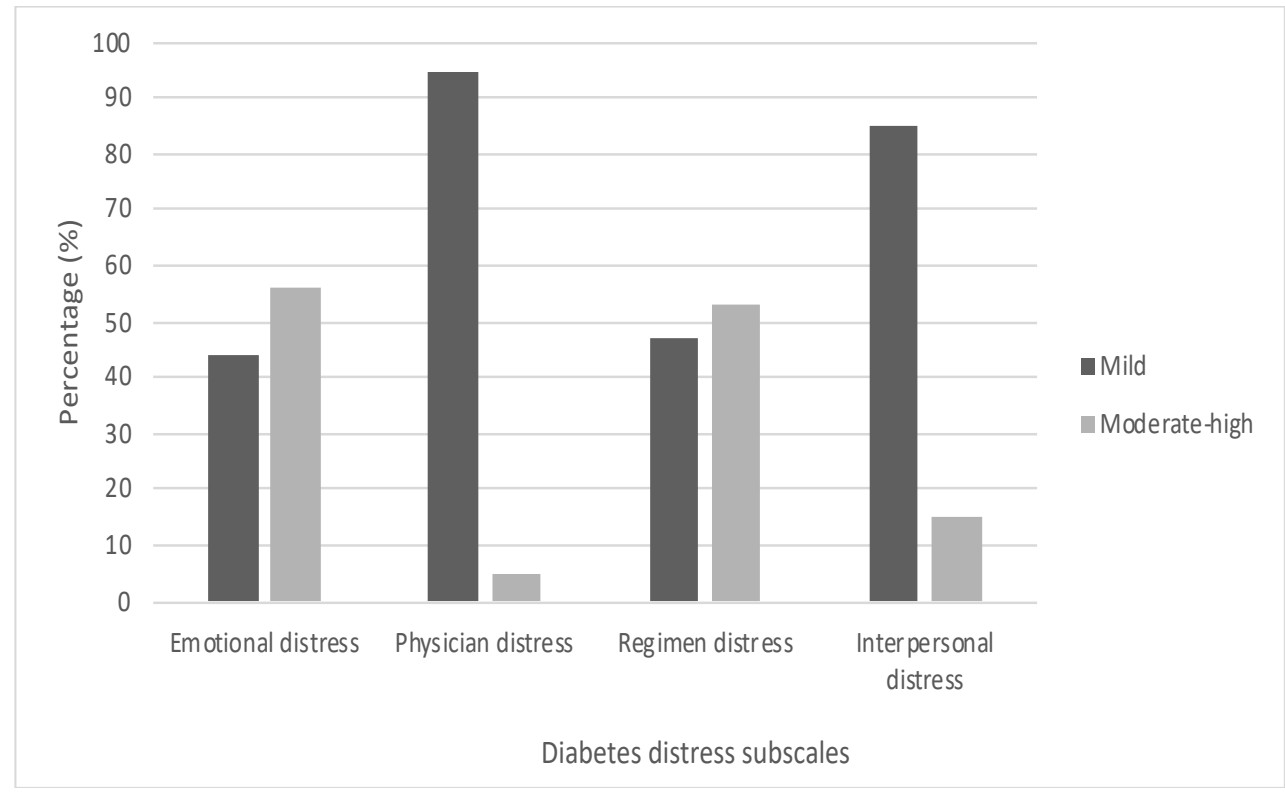

Figure 1. Percentage of diabetes distress based on subscales

Table 2. Associations of patients' sociodemographic with diabetes distress

\begin{tabular}{lccc}
\hline \multirow{2}{*}{ Characteristic } & \multicolumn{2}{c}{ Diabetes Distress } & \multirow{2}{*}{ P value } \\
\cline { 2 - 3 } Gender & $17(25.8)$ & Moderate & \\
$\quad$ Male & $49(74.2)$ & $30(78.9)$ & 0,641 \\
Female & & & \\
Age in years & $1(1.6)$ & $7(18.4)$ & $0,014^{*}$ \\
$\leq 49$ & $19(29.7)$ & $6(15.8)$ & \\
$50-59$ & $28(43.8)$ & $16(42.1)$ & \\
$60-69$ & $16(25.0)$ & $9(23.7)$ & \\
$\geq 70$ & & & \\
Type of insurance & $22(33.3)$ & $22(57.9)$ & $0,012^{*}$ \\
BPJS-PBI & $36(54.5)$ & $16(42.1)$ & \\
BPJS-Non PBI & $8(12.1)$ & 0 & \\
Without insurance & & & \\
Last education level & $19(29.7)$ & $12(31.6)$ & 0,780 \\
$\quad$ No formal education/ & & & \\
elementary school & $16(25.0)$ & $9(23.7)$ & \\
Junior high school & $22(34.4)$ & $15(39.5)$ & \\
Senior high school & $7(10.9)$ & $2(5.3)$ & \\
$\quad$ University & & & \\
Time from diagnosis, years & $3.8(4.0)$ & $4.4(3.8)$ & 0,289 \\
Diabetes, mean (SD) & $5.4(5.7)$ & $4.6(5.1)$ & 0,285 \\
Hypertension, mean (SD) & & & \\
\hline
\end{tabular}

Abbreviations: BPJS-PBI: patients who could not afford to pay the health insurance premium, BPJS-Non PBI: patients who afford to pay the health insurance premium, SD: standard deviation. $*$ Statistically significant difference $(\mathrm{p}<0.05)$ using Pearson $\chi^{2}$ and Mann-Whitney tests

Prevalence of diabetes... (Alfian et al.,) 
The strength of our study is that our population consists of T2DM patients with hypertension as comorbidities, as it is representative of T2DM patients. In addition, the high response rate reported in our study indicates our findings are generalizable for T2DM patients who visit the CHCs in Bandung City, Indonesia. However, our study might underestimate the level of diabetes distress because of social desirability and recall bias. Furthermore, most of the patients participated in our study were those who regularly visited the $\mathrm{CHCs}$, which is reflected with most patients experiencing mild diabetes distress. Studies evaluating the association between sociodemographic factors and diabetes distress in less controlled T2DM patients using antihypertensive medications are therefore needed.

Our findings highlight a need for a routine diabetes distress assessment among T2DM patients with comorbidity in primary care settings, particularly for the elderly and patients who could not afford to pay the health insurance premium T2DM patients who experienced moderate-high diabetes distress may need early and additional supports from health care providers to address these specific areas of diabetes management.

\section{CONCLUSION}

Emotional and regimen distress as a dominant forms of diabetes distress was observed among T2DM patients using antihypertensive medications. A routine diabetes distress assessment is needed in T2DM patients with comorbidity in primary care settings, particularly for elderly and patients who could not afford to pay the health insurance premium.

\section{ACKNOWLEDGEMENT}

We thank all research assistants and participants for their contribution.

\section{REFERENCES}

Abdulbari Bener, A. O. A. A. A.-H. and E. E. D. (2011). High prevalence of depression, anxiety and stress symptoms among diabetes mellitus patients. The Open Psychiatry Journal, 5, 5-12

Almeda, J. ., Capistrano, T. ., \& Sarte, G. . (2010). Elementary Statistic. University of The Philipines Press.

American Diabetes Association. (2019a). 5. Lifestyle management: Standards of medical care in diabetesd2019. Diabetes Care, 42(January), S46-S60. https://doi.org/10.2337/dc19-S005

American Diabetes Association. (2019b). Standards of Medical Care in. Clinical Diabetes Journal, 42, $1-194$

Arifin, B., Perwitasari, D. A., Thobari, J. A., Cao, Q., Krabbe, P. F. M., \& Postma, M. J. (2017). Translation, Revision, and Validation of the Diabetes Distress Scale for Indonesian Type 2 Diabetic Outpatients with Various Types of Complications. In Value in Health Regional Issues (Vol. 12, pp. 63-73). https://doi.org/10.1016/j.vhri.2017.03.010

Arifin, B., van Asselt, A. D. I., Setiawan, D., Atthobari, J., Postma, M. J., \& Cao, Q. (2019). Diabetes distress in Indonesian patients with type 2 diabetes: a comparison between primary and tertiary care. BMC Health Services Research, 19:773, 1-11. https://doi.org/10.1186/s12913-019-4515-1

Baek, R. N., Tanenbaum, M. L., \& Gonzalez, J. S. (2014). Diabetes Burden and Diabetes Distress: the Buffering Effect of Social Support. Annals of Behavioral Medicine, 48(2), 145-155. https://doi.org/10.1007/s12160-013-9585-4

Bruno, B. A., Choi, D., Thorpe, K. E., \& Yu, C. H. (2019). Relationship among diabetes distress, decisional conflict, quality of life, and patient perception of chronic illness care in a cohort of patients with type 2 diabetes and other comorbidities. Diabetes Care, 42(7), 1170-1177. https://doi.org/10.2337/dc18-1256

Carls, G., Huynh, J., Tuttle, E., Yee, J., \& Edelman, S. V. (2017). Achievement of Glycated Hemoglobin Goals in the US Remains Unchanged Through 2014. Diabetes Therapy, 8(4), $863-$ 873. https://doi.org/10.1007/s13300-017-0280-5 
Chew, B. H., Mohd-Sidik, S., \& Shariff-Ghazali, S. (2015). Negative effects of diabetes-related distress on health-related quality of life: An evaluation among the adult patients with type 2 diabetes mellitus in three primary healthcare clinics in Malaysia. Health and Quality of Life Outcomes, 13(1),1-45. https://doi.org/10.1186/s12955-015-0384-4

Chew, B. H., Vos, R., Mohd-Sidik, S., \& Rutten, G. E. H. M. (2016). Diabetes-Related distress, depression and Distress-Depression among adults with type 2 diabetes mellitus in Malaysia. PLoS ONE, 11(3), 1-16. https://doi.org/10.1371/journal.pone.0152095

Chipidza, F. E., Wallwork, R. S., \& Stern, T. A. (2015). Impact of the doctor-patient relationship. Primary Care Companion to the Journal of Clinical Psychiatry, 17(5),1-18. https://doi.org/10.4088/PCC.15f01840

Dennick, K., Sturt, J., Hessler, D., Purssell, E., Hunter, B., Oliver, J., \& Fisher, L. (2015). High rates of elevated diabetes distress in research populations: A systematic review and meta-analysis. International Diabetes Nursing, 12(3), 93-107. https://doi.org/10.1080/20573316.2016.1202497

Emre, N., Topal, K., Edirne, T., \& Gereklioğlu, Ç. (2018). Factors affecting risk of anxiety and depression among diabetic and hypertensive patients who refer to family health centers. International Journal of Diabetes in Developing Countries, 38(3), 305-311. https://doi.org/10.1007/s13410-017-0592-z

Interntional Diabetes Federation. (2017). IDF Diabetes Atlas. https://www.bing.com/search?q=International+Diabetes+Federation+(IDF).+(2017).+IDF+Diabe tes+Atlas.+Retrieved+from+http\%3A\%2F\%2Fwww.diabetesatlas.org\%2Fresources\%2F2017atlas.html\&cvid=df501 fbc7d1249f69cfae68e1c8be7aa\&aqs=edge..69i57.1093j0j1\&pglt=2083\& FOR

Fisher, E. B., Thorpe, C. T., Devellis, B. M. E., \& Devellis, R. F. (2007). Healthy coping, negative emotions, and diabetes management: A systematic review and appraisal. Diabetes Educator, 33(6), 1080-1103. https://doi.org/10.1177/0145721707309808

Fisher, L., Hessler, D. M., Polonsky, W. H., \& Mullan, J. (2012). When is diabetes distress clinically meaningful? Establishing cut points for the diabetes distress scale. Diabetes Care, 35(2), 259264. https://doi.org/10.2337/dc11-1572

Fisher, L., Mullan, J. T., Arean, P., Glasgow, R. E., Hessler, D., \& Masharani, U. (2010). Diabetes distress but not clinical depression or depressive symptoms is associated with glycemic control in both cross-sectional and longitudinal analyses. Diabetes Care, 33(1), 23-28. https://doi.org/10.2337/dc09-1238

Fisher, L., Mullan, J. T., Skaff, M. M., Glasgow, R. E., Arean, P., \& Hessler, D. (2009). Predicting diabetes distress in patients with Type 2 diabetes: A longitudinal study. Diabetic Medicine, 26(6), 622-627. https://doi.org/10.1111/j.1464-5491.2009.02730.x

Gahlan, D., Rajput, R., Gehlawat, P., \& Gupta, R. (2018). Prevalence and determinants of diabetes distress in patients of diabetes mellitus in a tertiary care centre. Diabetes and Metabolic Syndrome: Clinical Research and Reviews, 12(3), 333-336. https://doi.org/10.1016/j.dsx.2017.12.024

Hannonen, R., Eklund, K., Tolvanen, A., Komulainen, J., Riikonen, R., Delamater, A. M., \& Ahonen, T. (2015). Psychological distress of children with early-onset type 1 diabetes and their mothers' well-being. Acta Paediatrica, International Journal of Paediatrics, 104(11), 1144-1149. https://doi.org/10.1111/apa.13144

Naicker, K., Johnson, J. A., Skogen, J. C., Manuel, D., Øverland, S., Sivertsen, B., \& Colman, I. (2017). Type 2 diabetes and comorbid symptoms of depression and anxiety: Longitudinal associations with mortality risk. Diabetes Care, 40(3), 352-358. https://doi.org/10.2337/dc16$\underline{2018}$

Ogurtsova, K., da Rocha Fernandes, J. D., Huang, Y., Linnenkamp, U., Guariguata, L., Cho, N. H., Cavan, D., Shaw, J. E., \& Makaroff, L. E. (2017). IDF Diabetes Atlas: Global estimates for the prevalence of diabetes for 2015 and 2040. Diabetes Research and Clinical Practice, 128, 40-50. 
https://doi.org/10.1016/j.diabres.2017.03.024

Polonsky, W. H., Fisher, L., Earles, J., James Dude, R., Lees, J., Mullan, J., \& Jackson, R. A. (2005). Assessing Psychosocial Distress in. Diabetes Care, 28(3), 626-631

Rubin, R. R., \& Peyrot, M. (1999). Quality of life and diabetes. Diabetes/Metabolism Research and Reviews, 15(3), 205-218. https://doi.org/10.1002/(SICI)1520-7560(199905/06)15:3<205::AIDDMRR29>3.0.CO;2-O

Sturt, J., Dennick, K., Hessler, D., Hunter, B. M., Oliver, J., \& Fisher, L. (2015). Effective interventions for reducing diabetes distress: systematic review and meta-analysis. International Diabetes Nursing, 12(2), 40-55. https://doi.org/10.1179/2057332415y.0000000004

Wardian, J., \& Sun, F. (2014). Factors Associated With Diabetes-Related Distress: Implications for Diabetes Self-Management. Social Work in Health Care, 53(4), 364-381. https://doi.org/10.1080/00981389.2014.884038

Zanchetta, F. C., Trevisan, D. D., Apolinario, P. P., Silva, J. B. da, \& Lima, M. H. de M. (2016). Clinical and sociodemographic variables associated with diabetes-related distress in patients with type 2 diabetes mellitus. Einstein (Sao Paulo, Brazil), 14(3), 346-351. https://doi.org/10.1590/S1679-45082016AO3709 